\title{
Generalized Convolution Roots of Positive Definite Kernels on Complex Spheres
}

\author{
Victor S. BARBOSA and Valdir A. MENEGATTO \\ Instituto de Ciências Matemáticas e de Computação, Universidade de São Paulo, \\ Caixa Postal 668, 13560-970, São Carlos - SP, Brasil \\ E-mail:victorrsb@gmail.com, menegatt@icmc.usp.br
}

Received October 16, 2014, in final form February 10, 2015; Published online February 13, 2015 http://dx.doi.org/10.3842/SIGMA.2015.014

\begin{abstract}
Convolution is an important tool in the construction of positive definite kernels on a manifold. This contribution provides conditions on an $L^{2}$-positive definite and zonal kernel on the unit sphere of $\mathbb{C}^{q}$ in order that the kernel can be recovered as a generalized convolution root of an equally positive definite and zonal kernel.
\end{abstract}

Key words: positive definiteness; zonal kernels; recovery formula; convolution roots; Zernike or disc polynomials

2010 Mathematics Subject Classification: 33C55; 41A35; 41A63; 42A82; 42A85

\section{Introduction}

In this paper, we deal with a specific problem involving the so-called positive definite kernels. These kernels have importance in the resolution of issues pertaining to many areas of mathematics, including approximation theory, functional analysis, statistics, etc, presenting themselves in both theory and applications. The concept of positive definiteness usually appears in two formats as we now introduce.

Let $X$ be a nonempty set and $K$ a kernel on $X$, that is, a complex function $K$ with domain $X \times X$. The kernel $K$ is positive definite if

$$
\sum_{m, n=1}^{k} c_{m} \overline{c_{n}} K\left(x_{m}, x_{n}\right) \geq 0,
$$

for all positive integer $k$, all subsets $\left\{x_{1}, x_{2}, \ldots, x_{k}\right\}$ of $X$ and any complex numbers $c_{1}, c_{2}, \ldots, c_{k}$. As for the second concept of positive definiteness, we need to assume two facts: $X$ is endowed with a measure $\mu$ so that $(X, \mu)$ is a $\sigma$-finite measure space and the kernel $K$ needs to be an element of $L^{2}(X \times X, \mu \times \mu)$. As usual, we will speak of the elements in this space as if they were functions, with equality interpreted as equality $\mu$-a.e.. A kernel $K$ in $L^{2}(X \times X, \mu \times \mu)$ is $L^{2}$-positive definite if

$$
\int_{X}\left(\int_{X} K(x, y) f(y) d \mu(y)\right) \overline{f(x)} d \mu(x) \geq 0, \quad f \in L^{2}(X, \mu) .
$$

If $\mathcal{K}$ is the integral operator on $L^{2}(X, \mu)$ generated by $K$, the previous inequality corresponds to $\langle\mathcal{K} f, f\rangle_{2} \geq 0, f \in L^{2}(X, \mu)$, in which $\langle\cdot, \cdot\rangle_{2}$ is the usual inner product of $L^{2}(X, \mu)$.

In the cases in which the setting allows a comparison between the two concepts, it is not hard to see that they do not coincide. If one can speak of continuity in $X$, then a chance of coincidence increases considerably (see [3] for additional information on a possible equivalence). In this paper, we prefer to deal almost exclusively with $L^{2}$-positive definiteness since the source of the main problem to be discussed comes from functional analysis. 
If an $L^{2}$-positive definite kernel $K$ from $L^{2}(X \times X, \mu \times \mu)$ is available, a quite general problem is the determination of a kernel $S$ in $L^{2}(X \times X, \mu \times \mu)$ satisfying the integral relation

$$
\int_{X} S(x, \xi) S(\xi, y) d \mu(\xi)=K(x, y), \quad x, y \in X .
$$

If existence is guaranteed, the uniqueness of $S$ cannot be usually reached unless additional requirements are added (indeed, if $S$ is a solution, then $-S$ is another one).

In [3], the existence of a positive definite solution for this general problem was solved in the case in which $X$ is a metric space, the measure $\mu$ is strictly positive and $K$ is continuous. The solution $S$ itself was constructed via the square root $\mathcal{K}^{1 / 2}$ of the integral operator $\mathcal{K}$ acting on $L^{2}(X, \mu)$ and generated by $K$. As a matter of fact, the operator $\mathcal{K}^{1 / 2}$ was an integral operator on $L^{2}(X, \mu)$ itself and the generating kernel of $\mathcal{K}^{1 / 2}$ was a solution $S$, equally continuous and positive definite. The results proved in [3] agree with those previously surveyed in [13], the main difference between them being the setting considered in each case.

If $X$ has an enhanced structure, the original kernel $K$ may have additional and desirable features. Hence, the problem specializes to the following version: is there a solution kernel $S$ matching all the relevant additional properties the original kernel $K$ may have? In some cases, the left-hand side of (1.2) generalizes the notion of convolution. For instance, if $X$ is a compact two-point homogeneous manifold and $\mu$ is the usual "volume" measure on $X$, then the integral in (1.2) is an extension of the concept of spherical convolution described in $[9,10]$ when the kernel $S$ is zonal (isotropic). In particular, the solution of the proposed problem boils down to the finding of a convolution root $S$ for $K$ having the same features as $K$. This is relevant on itself because (spherical) convolution is known to be an efficient tool in the construction of positive definite kernels. We also observe that the case in which $X$ is a sphere, is of particular relevancy in statistics and approximation theory (see $[2,14,19])$.

Among the many research problems collected in [4] the readers can find open questions aligned with the material covered in the references mentioned in the previous paragraph and with the results to be developed in Sections 2 and 3 ahead. In those sections, we will chase a solution for (1.2) in the case in which $X$ is the unit sphere $\Omega_{2 q}$ in $\mathbb{C}^{q}, \mu$ is the usual Lebesgue measure in $\Omega_{2 q}$, and $K$ is zonal, following the direct procedure adopted in [19], a method that contemplates a Fourier analysis perspective. The procedure itself encompasses these steps: generalized convolutions preserve zonality; zonal kernels on $\Omega_{2 q}$ have specific expansions that are necessary and sufficient for zonality; generalized convolution acts on these expansions by squaring coefficients; the existence of zonal generalized convolution square roots is a question of convergence of expansions; starting from general zonal kernels, one step of generalized convolution generates a continuous zonal kernel. In particular, it should become clear to the reader that the paper deals with a standard argument that features an operation (generalized convolution), a symmetry property (zonality), and a representation (series expansion) that maintains the symmetry and is invariant under the operation.

We believe the approach developed here can be adapted to many other situations, a typical example being some compact two-point homogeneous manifolds. The setting and the notion of generalized spherical convolution used in the paper, can be found in [6], where a multiplier version of the Bernstein inequality on $\Omega_{2 q}$ was obtained. In order to direct the reader for potential applications involving the analysis on $\Omega_{2 q}$ and perhaps, to the specific material covered here, we mention $[16,17]$.

The spherical approach taken in the paper is probably less general than that adopted in the Hilbert-Schmidt theory (that was the option in [3, 13]). For the convenience of the reader and also for possible comparisons, that more general approach will be sketched in Section 4 . However, it is valuable to mention that the technique adopted here is more explicit and allows the study of regularity properties of the convolution roots and of the kernel. 


\section{The spherical case plus zonality}

Let $\sigma_{q}$ denote the surface measure on the unit sphere $\Omega_{2 q}$ of $\mathbb{C}^{q}$. Assuming $K$ is a zonal and $L^{2}$-positive definite kernel on $\Omega_{2 q}$, we will seek for a kernel $S$ in $L^{2}\left(\Omega_{2 q} \times \Omega_{2 q}, \sigma_{q} \times \sigma_{q}\right)$, of the same type as $K$, so that

$$
\int_{\Omega_{2 q}} S(x, \xi) S(\xi, y) d \sigma_{q}(\xi)=K(x, y), \quad x, y \in \Omega_{2 q} .
$$

Classical results from the analysis on the sphere $\Omega_{2 q}$ and also basic Fourier analysis on $\Omega_{2 q}$ will be needed along the way. In more than half of this section, we will detail some of them and will prove some others not available in the literature. Recent references related to the material to be described in this section are [1, 18] while classical ones are $[5,12]$.

The measure $\sigma_{q}$ is invariant with respect to the group $\mathcal{O}(2 q)$ of all unitary linear operators on $\mathbb{C}^{q}$ in the following sense: if $E$ is a $\sigma_{q}$-measurable subset of $\Omega_{2 q}$ and $\rho$ is an element of $\mathcal{O}(2 q)$ then $\sigma_{q}(\rho(E))=\sigma_{q}(E)$. Zonality of a kernel $K$ refers to the invariance property

$$
K(\rho(x), \rho(y))=K(x, y), \quad x, y \in \Omega_{2 q}, \quad \rho \in \mathcal{O}(2 q) .
$$

We simplify notation by writing $L^{2}\left(\Omega_{2 q}\right):=L^{2}\left(\Omega_{2 q}, \sigma_{q}\right), \Omega_{2 q}^{2}:=\Omega_{2 q} \times \Omega_{2 q}$ and $L^{2}\left(\Omega_{2 q}^{2}\right):=$ $L^{2}\left(\Omega_{2 q}^{2}, \sigma_{q} \times \sigma_{q}\right)$. The pertinent inner products in these Hilbert spaces will appear in normalized form, that is,

$$
\langle f, g\rangle_{2}:=\frac{1}{\omega_{q}} \int_{\Omega_{2 q}} f(x) \overline{g(x)} d \sigma_{q}(x), \quad f, g \in L^{2}\left(\Omega_{2 q}\right),
$$

and

$$
\left\langle K_{1}, K_{2}\right\rangle_{2}:=\frac{1}{\omega_{q}^{2}} \int_{\Omega_{2 q}^{2}} K_{1}(x, y) \overline{K_{2}(x, y)} d\left(\sigma_{q} \times \sigma_{q}\right)(x, y), \quad K_{1}, K_{2} \in L^{2}\left(\Omega_{2 q}^{2}\right),
$$

respectively, in which $\omega_{q}:=2 \pi^{q} /(q-1)$ ! is the surface area of $\Omega_{2 q}$.

The generalized convolution of two kernels $K_{1}$ and $K_{2}$ from $L^{2}\left(\Omega_{2 q}^{2}\right)$ is the kernel $K_{1} * K_{2}$ given by the formula

$$
\left(K_{1} * K_{2}\right)(x, y)=\frac{1}{\omega_{q}} \int_{\Omega_{2 q}} K_{1}(x, \xi) K_{2}(\xi, y) d \sigma_{q}(\xi), \quad x, y \in \Omega_{2 q} .
$$

Clearly, the definition makes sense as long as the integral is well-defined.

From now on, in order to make the presentation clearer, we need to consider two separate cases. For $q \geq 2$, the zonality of $K$ corresponds to the existence of a function $K^{\prime}: B[0,1] \rightarrow \mathbb{C}$ so that

$$
K(x, y)=K^{\prime}(x \cdot y), \quad x, y \in \Omega_{2 q},
$$

in which - denotes the usual inner product in $\mathbb{C}^{q}$ and $B[0,1]:=\{z \in \mathbb{C}: z \bar{z} \leq 1\}$. Thus, if $K_{2}$ is zonal and we replace $K_{1}$ with a function $f$ that depends upon $\xi$ only, then the convolution $K_{1} * K_{2}$ becomes the spherical convolution of $K_{2}^{\prime}$ and $\bar{f}$ as defined in [7, 11].

Adapting arguments found in [8], one can see that a kernel $K$ on $\Omega_{2 q}$ is $L^{2}$-positive definite and zonal if and only if the generating function $K^{\prime}$ appearing in (2.2) have a double series representation of the form

$$
K^{\prime}(z)=\sum_{m, n=0}^{\infty} a_{m, n}^{q-2}\left(K^{\prime}\right) R_{m, n}^{q-2}(z), \quad z \in B[0,1],
$$


in which $a_{m, n}^{q-2}\left(K^{\prime}\right) \geq 0, m, n \in \mathbb{Z}_{+}$, with convergence in $L^{2}\left(B[0,1], \nu_{q-2}\right)$, where

$$
d \nu_{q-2}(z)=\frac{q-1}{\pi}\left(1-|z|^{2}\right)^{q-2} d x d y, \quad z \in B[0,1] .
$$

The symbol $R_{m, n}^{q-2}$ stands for the disk or generalized Zernike polynomial of bi-degree $(m, n)$ associated with the dimension $q$, that is,

$$
R_{m, n}^{q-2}(z):=r^{|m-n|} e^{i(m-n) \theta} R_{m \wedge n}^{(q-2,|m-n|)}\left(2 r^{2}-1\right), \quad z=r e^{i \theta} \in B[0,1],
$$

where $R_{m \wedge n}^{(q-2,|m-n|)}$ is the Jacobi polynomial of degree $m \wedge n:=\min \{m, n\}$ associated with the pair of numbers $q-2$ and $|m-n|$, normalized so that $R_{m \wedge n}^{(q-2,|m-n|)}(1)=1$. Since

$$
R_{k}^{(q-2,|m-n|)}=\frac{P_{k}^{(q-2,|m-n|)}}{P_{k}^{(q-2,|m-n|)}(1)}
$$

in which $P_{k}^{(q-2,|m-n|)}$ is the classical Jacobi polynomial of degree $k$ associated with $q-2$ and $|m-n|$, as defined in [15], then $\left|R_{m, n}^{q-2}(z)\right| \leq 1, z \in B[0,1]$ and $R_{m, n}^{q-2}(1)=1$ for all $m$ and $n$. An alternative formula for $R_{m, n}^{q-2}$ via standard monomials is

$$
R_{m, n}^{q-2}(z)=\frac{m ! n !(q-2) !}{(m+q-2) !(n+q-2) !} \sum_{j=0}^{m \wedge n} \frac{(-1)^{j}(m+n+q-2-j) !}{j !(m-j) !(n-j) !} z^{m-j} \bar{z}^{n-j}
$$

The set $\left\{R_{m, n}^{q-2}: m, n \in \mathbb{Z}_{+}\right\}$is an orthogonal system in $L^{2}\left(B[0,1], \nu_{q-2}\right)$, that is,

$$
\int_{B[0,1]} R_{m, n}^{q-2}(z) \overline{R_{k, l}^{q-2}(z)} d \nu_{q-2}(z)=\frac{\delta_{m k} \delta_{n l}}{h_{m, n}^{q-2}}
$$

where

$$
h_{m, n}^{q-2}=\frac{m+n+q-1}{q-1}\left(\begin{array}{c}
m+q-2 \\
q-2
\end{array}\right)\left(\begin{array}{c}
n+q-2 \\
q-2
\end{array}\right) .
$$

If $K$ is a zonal kernel on $\Omega_{2 q}$, we will write $a_{m, n}^{q-2}\left(K^{\prime}\right)$ to denote the Fourier coefficient of the generating function $K^{\prime}$, with respect to the orthogonal basis $\left\{R_{m, n}^{q-2}: m, n \in \mathbb{Z}_{+}\right\}$of $L^{2}\left(B[0,1], \nu_{q-2}\right)$ :

$$
a_{m, n}^{q-2}\left(K^{\prime}\right)=h_{m, n}^{q-2} \int_{B[0,1]} K^{\prime}(z) \overline{R_{m, n}^{q-2}(z)} d \nu_{q-2}(z), \quad m, n \in \mathbb{Z}_{+} .
$$

The complex Funk-Hecke formula in $\Omega_{2 q}$ establishes an intimate connection among everything we have mentioned so far. If $Y$ is a spherical harmonic of bi-degree $(m, n)$ in $q$ dimensions, that is, $Y$ is the restriction to $\Omega_{2 q}$ of a polynomial of bidegree $(m, n)$ in $\mathbb{C}^{q}$ that belongs to the kernel of the complex Laplacian in $\mathbb{C}^{q}$, and $K^{\prime}$ is a function in $L^{2}\left(B[0,1], \nu_{q-2}\right)$, it states that $[7,11]$

$$
\frac{1}{\omega_{q}} \int_{\Omega_{2 q}} K^{\prime}(x \cdot y) \overline{Y(x)} d \sigma_{q}(x)=\left[\int_{B[0,1]} K^{\prime}(z) \overline{R_{m, n}^{q-2}(z)} d \nu_{q-2}(z)\right] \overline{Y(y)}, y \in \Omega_{2 q},
$$

as long as the integral on the left-hand side exists. In particular, the Funk-Hecke formula simplifies to

$$
\frac{1}{\omega_{q}} \int_{\Omega_{2 q}} K^{\prime}(x \cdot y) \overline{Y(x)} d \sigma_{q}(x)=\frac{a_{m, n}^{q-2}\left(K^{\prime}\right)}{h_{m, n}^{q-2}} \overline{Y(y)}, \quad y \in \Omega_{2 q} .
$$


We now move to the case $q=1$. The zonality of a kernel $K$ on $\Omega_{2}$ corresponds to the existence of a function $K^{\prime}: \Omega_{2} \rightarrow \mathbb{C}$ so that

$$
K(x, y)=K^{\prime}(x \cdot y), \quad x, y \in \Omega_{2},
$$

The characterization of the $L^{2}$-positive definite and zonal kernels on $\Omega_{2}$ skips a little bit from the previous representation in higher dimensions. In this particular case, the function $K^{\prime}$ appearing in (2.5) have a series representation of the form

$$
K^{\prime}(z)=\sum_{k=-\infty}^{\infty} a_{k}\left(K^{\prime}\right) z^{k}, \quad z \in \Omega_{2}
$$

in which $a_{k}\left(K^{\prime}\right) \geq 0, k \in \mathbb{Z}$, with convergence of the series in $L^{2}\left(\Omega_{2}\right)$. If one wants to speak of orthogonality, integration needs to be done in accordance with the standard Fourier theory, interpreting $\Omega_{2}$ as the quotient space $\mathbb{R} / 2 \pi \mathbb{Z}$, under the equivalence relation $x \sim y$ if and only if $x-y \in 2 \pi \mathbb{Z}$. If $q: \mathbb{R} \rightarrow \Omega_{2}$ is the quotient map, then (the invariant) integration on $\Omega_{2}$ reads like

$$
\int_{\Omega_{2}} f(x) d \sigma_{2}(x):=\int_{0}^{2 \pi} f(q(\theta)) d \theta
$$

in which $d \theta$ is the Lebesgue measure element on the interval $[0,2 \pi)$. In this sense, if

$$
R_{m}(z):=z^{m}, \quad z \in \Omega_{2}, \quad m \in \mathbb{Z},
$$

then $\left\{R_{m}: m \in \mathbb{Z}\right\}$ is an orthonormal basis of $L^{2}\left(\Omega_{2 q}, \sigma_{2}\right)$. Indeed, first observe that

$$
\frac{1}{\omega_{2}} \int_{\Omega_{2}} R_{m}(z) \overline{R_{n}(z)} d \sigma_{2}(z)=\frac{1}{\omega_{2}} \int_{0}^{2 \pi} e^{i(m-n) \theta} d \theta .
$$

As so, if $m=n$ then the integral on the right-hand side is just 1 . Otherwise, we can pick $\theta_{0} \in[0,2 \pi]$ so that $e^{i(m-n) \theta_{0}} \neq 1$ and use a change of variables to justify the equalities

$$
\frac{1}{\omega_{2}} \int_{0}^{2 \pi} e^{i(m-n) \theta} d \theta=\frac{1}{\omega_{2}} \int_{0}^{2 \pi} e^{i(m-n)\left(\theta+\theta_{0}\right)} d \theta=\frac{1}{\omega_{2}} e^{i(m-n) \theta_{0}} \int_{0}^{2 \pi} e^{i(m-n) \theta} d \theta,
$$

and arrive at

$$
\frac{1}{\omega_{2}} \int_{0}^{2 \pi} e^{i(m-n) \theta} d \theta=0
$$

To close the section, we will list some specific properties of the space

$$
\mathcal{B}^{2}\left(\Omega_{2 q}^{2}\right):=\left\{K \in L^{2}\left(\Omega_{2 q}^{2}\right): K \text { is zonal }\right\},
$$

some of them to be used ahead. The norm in the spaces $L^{2}\left(\Omega_{2 q}^{2}\right)$ will be written as $\|\cdot\|_{2}$. The first one is of general interest and is included for completeness only. If $\left\{K_{n}\right\}$ is a sequence in $\mathcal{B}^{2}\left(\Omega_{2 q}^{2}\right)$ converging to $K \in L^{2}\left(\Omega_{2 q}^{2}\right), \rho \in \mathcal{O}(2 q)$ and we write $\rho K$ to denote the kernel $(x, y) \in \Omega_{2 q} \times \Omega_{2 q} \rightarrow K(\rho x, \rho y)$, the zonality of each $K_{n}$ plus the invariance of $\sigma_{q}$ with respect to $\rho$ justify the inequality

$$
\begin{aligned}
0 & \leq\|K-\rho K\|_{2} \leq\left\|K-K_{n}\right\|_{2}+\left\|K_{n}-\rho K\right\|_{2} \\
& =\left\|K-K_{n}\right\|_{2}+\left\|\rho K_{n}-\rho K\right\|_{2}=2\left\|K-K_{n}\right\|_{2} .
\end{aligned}
$$


Letting $n \rightarrow \infty$ leads to $K=\rho K$ in $L^{2}\left(\Omega_{2 q}^{2}\right)$ and, consequently, we can conclude that $\mathcal{B}^{2}\left(\Omega_{2 q}^{2}\right)$ is a closed subspace of $L^{2}\left(\Omega_{2 q}^{2}\right)$.

To proceed, we will need two additional notations:

$$
Z_{m, n}(x, y):=R_{m, n}^{q-2}(x \cdot y), \quad m, n \in \mathbb{Z}_{+}, \quad x, y \in \Omega_{2 q}, \quad q \geq 2,
$$

and

$$
Z_{m}(x, y):=R_{m}(x \cdot y)=x^{m} y^{-m}, \quad m \in \mathbb{Z}, \quad x, y \in \Omega_{2} .
$$

Since $q$ will remain fixed, the omission of the dimension $q$ in both notations introduced above should cause no confusion.

In the proposition below, we establish orthogonality properties of the sets $\left\{Z_{m, n}: m, n \in \mathbb{Z}_{+}\right\}$ and $\left\{Z_{m}: m \in \mathbb{Z}\right\}$ in the Hilbert space $\left(\mathcal{B}^{2}\left(\Omega_{2 q}^{2}\right),\langle\cdot, \cdot\rangle_{2}\right)$.

Proposition 2.1. The following assertions hold:

(i) $(q \geq 2)$ the Hilbert space $\mathcal{B}^{2}\left(\Omega_{2 q}^{2}\right)$ is isometrically isomorphic to $L^{2}\left(B[0,1], \nu_{q-2}\right)$;

(ii) $(q \geq 2)$ the set $\left\{\left(h_{m, n}^{q-2}\right)^{1 / 2} Z_{m, n}: m, n \in \mathbb{Z}_{+}\right\}$is an orthonormal basis of $\mathcal{B}^{2}\left(\Omega_{2 q}^{2}\right)$;

(iii) the set $\left\{Z_{m}: m \in \mathbb{Z}\right\}$ is an orthonormal basis of $\mathcal{B}^{2}\left(\Omega_{2}^{2}\right)$.

Proof. Let us consider the case $q \geq 2$ first. If $K \in \mathcal{B}^{2}\left(\Omega_{2 q}^{2}\right)$, the Funk-Hecke formula implies that

$$
\begin{aligned}
\|K\|_{2}^{2} & =\frac{1}{\omega_{q}^{2}} \int_{\Omega_{2 q}^{2}} K^{\prime}(x \cdot y) \overline{K^{\prime}(x \cdot y)} d\left(\sigma_{q} \times \sigma_{q}\right)(x, y) \\
& =\int_{B[0,1]} K^{\prime}(z) \overline{K^{\prime}(z)} d \nu_{q-2}(z)=\left\|K^{\prime}\right\|_{2, q}^{2},
\end{aligned}
$$

in which $\|\cdot\|_{2, q}$ stands for the usual norm in the space $L^{2}\left(B[0,1], \nu_{q-2}\right)$. In particular, $K \in$ $\mathcal{B}^{2}\left(\Omega_{2 q}^{2}\right) \rightarrow K^{\prime} \in L^{2}\left(B[0,1], \nu_{q-2}\right)$ is an isometry. This takes care of (i). Since $\left\{R_{m, n}^{q-2}: m, n \in \mathbb{Z}_{+}\right\}$ is an orthogonal basis in $L^{2}\left(B[0,1], \nu_{q-2}\right)$, the polarization identity reveals that its inverse image by the isometry in $(i)$ is an orthogonal basis in $\mathcal{B}^{2}\left(\Omega_{2 q}^{2}\right)$. Recalling (2.4), the orthonormality of the set in (ii) follows. In order to see it is complete in $\mathcal{B}^{2}\left(\Omega_{2 q}^{2}\right)$, let $K \in \mathcal{B}^{2}\left(\Omega_{2 q}^{2}\right)$ and assume that

$$
\frac{1}{\omega_{q}^{2}} \int_{\Omega_{2 q}^{2}} K(x, y) \overline{R_{m, n}(x, y)} d\left(\sigma_{q} \times \sigma_{q}\right)(x, y)=0, \quad m, n \in \mathbb{Z}_{+} .
$$

Since that corresponds to

$$
\frac{1}{\omega_{q}} \int_{\Omega_{2 q}}\left(\frac{1}{\omega_{q}} \int_{\Omega_{2 q}} K^{\prime}(x \cdot y) R_{n, m}^{q-2}(x \cdot y) d \sigma_{q}(x)\right) d \sigma_{q}(y)=0, \quad m, n \in \mathbb{Z}_{+},
$$

an application of the Funk-Hecke formula leads to the equality

$$
\left(h_{m, n}^{q-2}\right)^{-1} a_{m, n}^{q-2}\left(K^{\prime}\right)=0, \quad m, n \in \mathbb{Z}_{+},
$$

that is,

$$
\int_{B[0,1]} K^{\prime}(z) \overline{R_{m, n}^{q-2}(z)} d \nu_{q-2}(z)=0, \quad m, n \in \mathbb{Z}_{+} .
$$


Since $\left\{R_{m, n}^{q-2}: m, n \in \mathbb{Z}_{+}\right\}$is an orthogonal basis of $L^{2}\left(B[0,1], \nu_{q-2}\right)$ and this space is complete, then $K^{\prime}=0$ in $L^{2}\left(B[0,1], \nu_{q-2}\right)$. In particular, $K=0$ in $\mathcal{B}^{2}\left(\Omega_{2 q}^{2}\right)$ and (ii) is proved. The orthonormality assertion in (iii) is clear. As for completeness, let $K \in \mathcal{B}^{2}\left(\Omega_{2}^{2}\right)$ and assume that

$$
\frac{1}{\omega_{2}^{2}} \int_{\Omega_{2}^{2}} K(x, y) \overline{Z_{m}(x, y)} d\left(\sigma_{2} \times \sigma_{2}\right)(x, y)=0, \quad m \in \mathbb{Z} .
$$

Since this equality is precisely

$$
\frac{1}{4 \pi^{2}} \int_{\Omega_{2}}\left(\int_{\Omega_{2}} K^{\prime}(x \cdot y) x^{-m} d \sigma_{2}(x)\right) y^{m} d \sigma_{2}(y)=0, \quad m, n \in \mathbb{Z},
$$

it follows that

$$
a_{m}\left(K^{\prime}\right) \int_{\Omega_{2}} y^{-m} y^{m} d \sigma_{2}(y)=0, \quad m \in \mathbb{Z},
$$

in which $a_{m}\left(K^{\prime}\right)$ is the $m$-th usual Fourier coefficient of $K^{\prime}$. In other words, $a_{m}\left(K^{\prime}\right)=0, m \in \mathbb{Z}$, that is, $K^{\prime}=0$.

We close the section detaching an obvious consequence of Proposition 2.1(ii).

Lemma $2.2(q \geq 2)$. If $K^{\prime}$ belongs to $L^{2}\left(B[0,1], \nu_{q-2}\right)$, then $\sum_{m, n=0}^{\infty}\left(h_{m, n}^{q-2}\right)^{-1} a_{m, n}^{q-2}\left(K^{\prime}\right)^{2}$ is convergent.

\section{Convolution roots in $\mathcal{B}^{2}\left(\Omega_{2 q}^{2}\right)$}

In this section, we finally analyze the problem described in the first paragraph of the previous section. The first steps provide technical results on the convolution operation $*$ defined in (2.1), in the case when one or both kernels are the basic elements of Proposition 2.1. The main results of the paper come right after that.

Lemma 3.1. The following properties hold:

(i) the convolution of a hermitian kernel from $L^{2}\left(\Omega_{2 q}^{2}\right)$ with itself is $L^{2}$-positive definite on $\Omega_{2 q}$;

(ii) if $K_{1}$ and $K_{2}$ belong to $\mathcal{B}^{2}\left(\Omega_{2 q}^{2}\right)$, then $K_{1} * K_{2}$ does so.

Proof. Write $\mathcal{K} * \mathcal{K}$ to denote the integral operator on $L^{2}\left(\Omega_{2 q}\right)$ generated by $K * K$. If $f \in$ $L^{2}\left(\Omega_{2 q}\right)$ and $K$ is a kernel on $\Omega_{2 q}$, then a double application of Fubini's theorem implies that

$$
\langle\mathcal{K} * \mathcal{K}(f), f\rangle_{2}=\frac{1}{\omega_{q}^{3}} \int_{\Omega_{2 q}} \int_{\Omega_{2 q}} K(x, \xi) \overline{f(x)} d \sigma_{q}(x) \int_{\Omega_{2 q}} K(\xi, y) f(y) d \sigma_{q}(y) d \sigma_{q}(\xi) .
$$

If $K$ is hermitian, the previous equality reduces itself to

$$
\langle\mathcal{K} * \mathcal{K}(f), f\rangle_{2}=\frac{1}{\omega_{q}} \int_{\Omega_{2 q}}\left|\frac{1}{\omega_{q}} \int_{\Omega_{2 q}} K(x, \xi) \overline{f(x)} d \sigma_{q}(x)\right|^{2} d \sigma_{q}(\xi) .
$$

This takes care of (i). As for (ii), if $\rho \in \mathcal{O}_{2 q}$, then

$$
\left(K_{1} * K_{2}\right)(\rho(x), \rho(y))=\frac{1}{\omega_{q}} \int_{\Omega_{2 q}} K_{1}\left(x, \rho^{*} \xi\right) K_{2}\left(\rho^{*} \xi, y\right) d \sigma_{q}(\xi), \quad x, y \in \Omega_{2 q},
$$

in which $\rho^{*}$ is the adjoint of $\rho$. Since the measure $\sigma_{q}$ is invariant with respect to elements of $\mathcal{O}_{2 q}$, it follows that

$$
\left(K_{1} * K_{2}\right)(\rho x, \rho y)=\frac{1}{\omega_{q}} \int_{\Omega_{2 q}} K_{1}\left(x, \rho^{*} \xi\right) K_{2}\left(\rho^{*} \xi, y\right) d \sigma_{q}\left(\rho^{*} \xi\right)=\left(K_{1} * K_{2}\right)(x, y), \quad x, y \in \Omega_{2 q},
$$

and $K$ is zonal. 
The lemma below intends to produce formulas for convolutions involving the basis elements of $\mathcal{B}^{2}\left(\Omega_{2 q}^{2}\right)$.

Lemma 3.2. The following formulas hold:

$$
\begin{aligned}
& h_{k, l}^{q-2}\left(Z_{m, n} * Z_{k, l}\right)=\delta_{k m} \delta_{l n} Z_{k, l}, \quad m, n, k, l \in \mathbb{Z}_{+}, \\
& Z_{m} * Z_{n}=\delta_{m, n} Z_{m}, \quad m, n \in \mathbb{Z} .
\end{aligned}
$$

Proof. In the case $q \geq 2$, an application of the Funk-Hecke formula leads to

$$
\begin{aligned}
\left(Z_{m, n} * Z_{k, l}\right)(x, y) & =\frac{1}{\omega_{q}} \int_{\Omega_{2 q}} R_{m, n}^{q-2}(x \cdot \xi) R_{k, l}^{q-2}(\xi \cdot y) d \sigma_{q}(\xi) \\
& =\frac{1}{\omega_{q}} \int_{\Omega_{2 q}} R_{k, l}^{q-2}(\xi \cdot y) \overline{R_{m, n}^{q-2}(\xi \cdot x)} d \sigma_{q}(\xi) \\
& =\frac{a_{m, n}^{q-2}\left(R_{k, l}^{q-2}\right)}{h_{m, n}^{q-2}} \overline{R_{m, n}^{q-2}(y \cdot x)}, \quad x, y \in \Omega_{2 q} .
\end{aligned}
$$

It is now clear that

$$
\left(Z_{m, n} * Z_{k, l}\right)(x, y)=\frac{\delta_{m k} \delta_{n l}}{h_{m, n}^{q-2}} R_{m, n}^{q-2}(x \cdot y), \quad x, y \in \Omega_{2 q} .
$$

Moving to the case $q=1$, direct computation shows that

$$
\begin{aligned}
\left(Z_{m} * Z_{n}\right)(x, y) & =\frac{1}{\omega_{2}} \int_{\Omega_{2}} Z_{m}(x, \xi) Z_{n}(\xi, y) d \sigma_{2}(\xi) \\
& =x^{m} y^{-n} \frac{1}{\omega_{2}} \int_{\Omega_{2}} \overline{R_{m}(\xi)} R_{n}(\xi) \sigma_{2}(\xi), \quad m, n \in \mathbb{Z}_{+}, \quad x, y \in \Omega_{2} .
\end{aligned}
$$

The use of the orthonormality of $\left\{R_{m}: m \in \mathbb{Z}\right\}$ in $L^{2}\left(\Omega_{2}\right)$ in the previous equation implies the second formula in the statement of the lemma.

Theorem 3.3. If $K$ belongs to $\mathcal{B}^{2}\left(\Omega_{2 q}^{2}\right)$ then

$$
K * Z_{m, n}=\frac{a_{m, n}^{q-2}\left(K^{\prime}\right)}{h_{m, n}^{q-2}} Z_{m, n}=Z_{m, n} * K, \quad m, n \in \mathbb{Z}_{+}, \quad q \geq 2,
$$

and

$$
K * Z_{m}=a_{m}\left(K^{\prime}\right) Z_{m}=Z_{m} * K, \quad m, n \in \mathbb{Z}_{+}, \quad q=1 .
$$

Proof. In the case $q \geq 2$, both equalities follow from calculations via the Funk-Hecke formula. As for the case $q=1$, it can be done by direct calculations and a change of variables.

The formulae in the theorem below are the key step towards the main results of this section.

Theorem 3.4. If $K$ belongs to $\mathcal{B}^{2}\left(\Omega_{2 q}^{2}\right)$ then

$$
\left\langle K * K, Z_{m, n}\right\rangle_{2}=\left[\frac{a_{m, n}^{q-2}\left(K^{\prime}\right)}{h_{m, n}^{q-2}}\right]^{2}, \quad m, n \in \mathbb{Z}_{+}, \quad q \geq 2,
$$

and

$$
\left\langle K * K, Z_{m}\right\rangle_{2}=a_{m}\left(K^{\prime}\right)^{2}, \quad m \in \mathbb{Z}, \quad q=1 .
$$


Proof. Fix $m, n \in \mathbb{Z}_{+}$. Introducing the equality from Lemma 3.2, we see that

$$
\begin{aligned}
\left\langle K * K, Z_{m, n}\right\rangle_{2} & =\frac{1}{\omega_{q}^{2}} \int_{\Omega_{2 q}} \int_{\Omega_{2 q}}(K * K)(\xi, \eta) Z_{m, n}(\eta, \xi) d \sigma_{q}(\xi) d \sigma_{q}(\eta) \\
& =\frac{h_{m, n}^{q-2}}{\omega_{q}^{2}} \int_{\Omega_{2 q}} \int_{\Omega_{2 q}}(K * K)(\xi, \eta)\left(Z_{m, n} * Z_{m, n}\right)(\eta, \xi) d \sigma_{q}(\xi) d \sigma_{q}(\eta) .
\end{aligned}
$$

Appealing to the definition of convolution and using Fubini's theorem twice to interchange integration orders, we deduce that

$$
\left\langle K * K, Z_{m, n}\right\rangle_{2}=\frac{h_{m, n}^{q-2}}{\omega_{q}^{2}} \int_{\Omega_{2 q}} \int_{\Omega_{2 q}}\left(K * Z_{m, n}\right)(x, y)\left(Z_{m, n} * K\right)(y, x) d \sigma_{q}(x) \sigma_{q}(y) .
$$

Applying Theorem 3.3, we obtain

$$
\left\langle K * K, Z_{m, n}\right\rangle_{2}=\left(h_{m, n}^{q-2}\right)^{-1} a_{m, n}^{q-2}\left(K^{\prime}\right)^{2}\left\|Z_{m, n}\right\|_{2}^{2}=\left(h_{m, n}^{q-2}\right)^{-1} a_{m, n}^{q-2}\left(K^{\prime}\right)^{2}\left\|R_{m, n}^{q-2}\right\|_{2, q}^{2},
$$

while (2.4) yields the first equality in the statement of the theorem. The other equality is proved in a similar manner.

Theorem 3.5. Let $K$ belong to $L^{2}\left(\Omega_{2 q}^{2}\right)$. If $K=J * J$ for some $J$ in $\mathcal{B}^{2}\left(\Omega_{2 q}^{2}\right)$, then

$$
\sum_{m, n=0}^{\infty} h_{m, n}^{q-2}\left|\left\langle K, Z_{m, n}\right\rangle_{2}\right|<\infty, \quad q \geq 2,
$$

and

$$
\sum_{m=-\infty}^{\infty}\left|\left\langle K, Z_{m}\right\rangle_{2}\right|<\infty, \quad q=1 .
$$

Proof. If $K=J * J$ with $J \in \mathcal{B}^{2}\left(\Omega_{2 q}^{2}\right)$, then Lemma 3.1 implies that $K \in \mathcal{B}^{2}\left(\Omega_{2 q}^{2}\right)$ as well. In the case $q \geq 2$, that allows an application of Theorem 3.4 to deduce the equality

$$
\left\langle K, Z_{m, n}\right\rangle_{2}=\left[\frac{a_{m, n}^{q-2}\left(J^{\prime}\right)}{h_{m, n}^{q-2}}\right]^{2}, \quad m, n \in \mathbb{Z}_{+},
$$

that is,

$$
h_{m, n}^{q-2}\left\langle K, Z_{m, n}\right\rangle_{2}=\left[\frac{a_{m, n}^{q-2}\left(J^{\prime}\right)}{\left(h_{m, n}^{q-2}\right)^{1 / 2}}\right]^{2}, \quad m, n \in \mathbb{Z}_{+} .
$$

Since $\left\{\left(h_{m, n}^{q-2}\right)^{1 / 2} R_{m, n}^{q-2}: m, n \in \mathbb{Z}^{+}\right\}$is an orthonormal basis of $L^{2}\left(B[0,1], \nu_{q-2}\right)$ and $J^{\prime} \in$ $L^{2}\left(B[0,1], \nu_{q-2}\right)$, we invoke Lemma 2.2 to conclude that

$$
\sum_{m, n=0}^{\infty} h_{m, n}^{q-2}\left|\left\langle K, Z_{m, n}\right\rangle_{2}\right|<\infty .
$$

The case $q=1$ can be handled in a similar manner.

Needless to say that, due to Lemma 3.1, the modulus sign in the convergence outcome of the previous theorem can be removed, as long as the kernel $J$ is hermitian.

Theorem 3.5 suggests a criterion for the existence of convolution roots, therefore, a solution to the problem described at the beginning of Section 2. 
Theorem 3.6. Let $K$ be a kernel in $L^{2}\left(\Omega_{2 q}^{2}\right)$. If $q \geq 2$, assume that all the Fourier coefficients $\left\langle K, Z_{m, n}\right\rangle_{2}$ are nonnegative and that

$$
\sum_{m, n=0}^{\infty} h_{m, n}^{q-2}\left\langle K, Z_{m, n}\right\rangle_{2}<\infty
$$

Otherwise, assume that all Fourier coefficients $\left\langle K, Z_{m}\right\rangle_{2}$ are nonnegative and that

$$
\sum_{m=-\infty}^{\infty}\left\langle K, Z_{m}\right\rangle_{2}<\infty
$$

Then, there exists an $L^{2}$-positive definite kernel $P$ in $\mathcal{B}^{2}\left(\Omega_{2 q}^{2}\right)$ such that $K=P * P$. In particular, $K$ is an $L^{2}$-positive definite element of $\mathcal{B}^{2}\left(\Omega_{2 q}^{2}\right)$.

Proof. We prove the theorem in the case $q \geq 2$ only. Let us consider the zonal kernel $P$ for which

$$
P^{\prime}=\sum_{m, n=0}^{\infty} h_{m, n}^{q-2}\left\langle K, Z_{m, n}\right\rangle_{2}^{1 / 2} Z_{m, n}
$$

The expansion of $P^{\prime}$ with respect to the orthonormal basis $\left\{\left(h_{m, n}^{q-2}\right)^{1 / 2} Z_{m, n}: m, n \in \mathbb{Z}_{+}\right\}$ of $\mathcal{B}^{2}\left(\Omega_{2 q}^{2}\right)$ is

$$
P^{\prime}=\sum_{m, n=0}^{\infty}\left(h_{m, n}^{q-2}\right)^{1 / 4}\left\langle K,\left(h_{m, n}^{q-2}\right)^{1 / 2} Z_{m, n}\right\rangle_{2}^{1 / 2}\left(h_{m, n}^{q-2}\right)^{1 / 2} Z_{m, n}, \quad m, n \in \mathbb{Z}_{+} .
$$

In particular, our convergence assumption implies that $P^{\prime}$ belongs to $\mathcal{B}^{2}\left(\Omega_{2 q}^{2}\right)$. Our additional assumptions and the characterization provided in (2.3) implies that $P$ is $L^{2}$-positive definite. At last, a help of Theorem 3.4 leads to

$$
\left\langle P * P, Z_{m, n}\right\rangle_{2}=\left[\frac{a_{m, n}^{q-2}\left(P^{\prime}\right)}{h_{m, n}^{q-2}}\right]^{2}=\left\langle K, Z_{m, n}\right\rangle_{2}, \quad m, n \in \mathbb{Z}_{+} .
$$

This suffices to conclude that $P * P=K$ in $L^{2}\left(\Omega_{2 q}^{2}\right)$.

The next result reveals that, in the setting we have adopted, the existence of a zonal convolution root of the kernel implies the existence of a continuous one.

Proposition 3.7. Let $K$ belong to $L^{2}\left(\Omega_{2 q}^{2}\right)$. If $K=J * J$ for some $J$ in $\mathcal{B}^{2}\left(\Omega_{2 q}^{2}\right)$, then $K$ is in fact a continuous kernel.

Proof. We know already that $K \in \mathcal{B}^{2}\left(\Omega_{2 q}^{2}\right)$. Hence, it can be written as

$$
K(x, y)=\sum_{m, n=0}^{\infty} a_{m, n}^{q-2}\left(K^{\prime}\right) R_{m, n}^{q-2}(x \cdot y) .
$$

Since $a_{m, n}^{q-2}=h_{m, n}^{q-2}\left\langle K, Z_{m, n}\right\rangle_{2}, m, n \in \mathbb{Z}_{+}$, it follows that

$$
\sum_{m, n=0}^{\infty}\left|a_{m, n}^{q-2}\left(K^{\prime}\right) R_{m, n}^{q-2}(x \cdot y)\right| \leq \sum_{m, n=0}^{\infty} h_{m, n}^{q-2}\left|\left\langle K, Z_{m, n}\right\rangle_{2}\right|, \quad x, y \in \Omega_{2 q} .
$$

Recalling Theorem 3.5, we can apply the Weierstrass M-test to conclude that the series in (3.1) converges uniformly in $\Omega_{2 q}^{2}$. Since each $R_{m, n}$ is continuous, it follows that the same series defines a continuous kernel. 
The next result is a consequence of the previous proposition and Theorem 2.3 in [3].

Corollary 3.8. Let $K$ belong to $L^{2}\left(\Omega_{2 q}^{2}\right)$. If $K=J * J$ for some $J$ in $\mathcal{B}^{2}\left(\Omega_{2 q}^{2}\right)$, then $K$ is positive definite in the usual sense.

This a simplified version of Theorem 3.6.

Theorem 3.9. If $K$ is a continuous, zonal and $L^{2}$-positive definite kernel on $\Omega_{2 q}$, then there exists an $L^{2}$-positive definite kernel $P$ in $\mathcal{B}^{2}\left(\Omega_{2 q}^{2}\right)$ such that $K=P * P$.

Proof. We only prove the assertion in the case $q \geq 2$. Write $K(x, y)=K^{\prime}(x \cdot y), x, y \in \Omega_{2 q}$, with $K^{\prime}$ as described in (2.3). Applying the Funk-Hecke formula, we see that

$$
\begin{aligned}
\left\langle K, Z_{m, n}\right\rangle_{2} & =\frac{1}{\omega_{q}^{2}} \int_{\Omega_{2 q}^{2}} K^{\prime}(x \cdot y) \overline{R_{m, n}^{q-2}(x \cdot y)} d\left(\sigma_{q} \times \sigma_{q}\right)(x, y) \\
& =\frac{1}{\omega_{q}} \frac{a_{m, n}^{q-2}\left(K^{\prime}\right)}{h_{m, n}^{q-2}} \int_{\Omega_{2 q}} \overline{R_{m, n}^{q-2}(y \cdot y)} d \sigma_{q}(y), \quad m, n \in \mathbb{Z}_{+},
\end{aligned}
$$

that is,

$$
\left\langle K, Z_{m, n}\right\rangle_{2}=\frac{a_{m, n}^{q-2}\left(K^{\prime}\right)}{h_{m, n}^{q-2}} \geq 0, \quad m, n \in \mathbb{Z}_{+} .
$$

Furthermore,

$$
\sum_{m, n=0}^{\infty} h_{m, n}^{q-2}\left\langle K, Z_{m, n}\right\rangle_{2}=\sum_{m, n=0}^{\infty} a_{m, n}^{q-2}\left(K^{\prime}\right)<\infty .
$$

An application of Theorem 3.6 justifies the assertion of the theorem.

We advise the reader that the results obtained in this section can be reproduced in some other important settings in both, real and complex versions. As a matter of fact, the approach can be developed as long as an $L^{2}$ structure similar to the one used here is available in the setting to be considered.

\section{Final remarks: the solution via integral operators}

Here, for the sake of completeness, we recall the functional analysis approach via HilbertSchmidt operator usually used to solve a version of the general recovery problem (1.2). One needs the separability of $L^{2}(X, \mu)$, the continuity of $K$ and, in addition, both concepts of positive definiteness need to coincide. In this case, (1.1) holds and the integral operator $\mathcal{K}$ generated by $K$ is a Hilbert-Schmidt operator.

The classical spectral and Mercer's theories guarantee the features below.

(i) The operator $\mathcal{K}$ is self-adjoint and Hilbert-Schmidt.

(ii) There exists a finite or countably infinite set of positive real numbers $\lambda_{1}(\mathcal{K}) \geq \lambda_{2}(\mathcal{K}) \geq$ $\cdots \geq 0$ and a corresponding orthonormal system $\left\{\phi_{n}\right\}$ of $L^{2}(X, \mu)$ so that

$$
\mathcal{K}\left(\phi_{n}\right)=\lambda_{n}(\mathcal{K}) \phi_{n}, \quad n=1,2, \ldots
$$

(iii) The sequence $\left\{\lambda_{n}(\mathcal{K})\right\}$ is square-summable and converges to 0 . 
(iv) The integral operator $\mathcal{K}$ has an $L^{2}$ representation in the form

$$
\mathcal{K}(f)=\sum_{n=1}^{\infty} \lambda_{n}(\mathcal{K})\left\langle f, \phi_{n}\right\rangle_{2} \phi_{n}, \quad f \in L^{2}(X) .
$$

(v) The kernel $K$ has an expansion in the form

$$
K(x, y)=\sum_{n=1}^{\infty} \lambda_{n}(\mathcal{K}) \phi_{n}(x) \overline{\phi_{n}(y)}
$$

with convergence in $L^{2}(X \times X, \mu \times \mu)$.

All this information being available, the following classical result holds.

Theorem 4.1. If $K$ is a continuous $L^{2}$-positive definite kernel on $X$, then there exists an $L^{2}$-positive definite kernel $S$ in $L^{2}(X \times X, \mu \times \mu)$, which can be taken continuous, so that

$$
\int_{X} S(x, \xi) S(\xi, y) d \mu(\xi)=K(x, y), \quad x, y \in X .
$$

The following result is also pertinent.

Corollary 4.2. Under the setting and the conditions stated in Theorem 4.1 , the square root $\mathcal{K}^{1 / 2}$ of $\mathcal{K}$ is the integral operator on $L^{2}(X, \mu)$ generated by $S$.

In this general context, the kernels $K$ and $S$ end up having the same $L^{2}$ expansion structure. Hence, it is expectable that $S$ will have the very same properties $K$ has, as long as the properties are attached to the Hilbert space structure of $L^{2}(X, \mu)$. In a certain sense, the results in Section 3 ratify this in the spherical setting, at least when the property to be preserved is zonality. We are unaware of specific papers addressing the analysis of a recovery property similar to the one considered here on $\Omega_{2 q}$ via this Functional Analysis approach.

\section{Acknowledgements}

The first author was partially supported by CAPES. The second one by FAPESP, under grant 2014/00277-5. Special thanks goes to the anonymous referees for the careful reading of the paper and for pointing corrections and suggestions that led to this final form of the paper.

\section{References}

[1] Bezubik A., Strasburger A., On spherical expansions of smooth SU(n)-zonal functions on the unit sphere in $\mathbb{C}^{n}$, J. Math. Anal. Appl. 404 (2013), 570-578, arXiv:1112.0648.

[2] Estrade A., Istas J., Ball throwing on spheres, Bernoulli 16 (2010), 953-970, arXiv:0810.4004.

[3] Ferreira J.C., Menegatto V.A., Eigenvalues of integral operators defined by smooth positive definite kernels, Integral Equations Operator Theory 64 (2009), 61-81.

[4] Gneiting T., Strictly and non-strictly positive definite functions on spheres, Bernoulli 19 (2013), 1327-1349, arXiv:1111.7077.

[5] Koornwinder T.H., The addition formula for Jacobi polynomials. II. The Laplace type integral representation and the product formula, Math. Centrum Amsterdam, Report TW133, 1976.

[6] Levesley J., Kushpel A., A multiplier version of the Bernstein inequality on the complex sphere, J. Comput. Appl. Math. 240 (2013), 184-191, arXiv:1204.6147.

[7] Menegatto V.A., Oliveira C.P., Annihilating properties of convolution operators on complex spheres, Anal. Math. 31 (2005), 13-30. 
[8] Menegatto V.A., Peron A.P., Positive definite kernels on complex spheres, J. Math. Anal. Appl. 254 (2001), 219-232.

[9] Platonov S.S., Approximations on compact symmetric spaces of rank 1, Sb. Math. 188 (1997), 753-769.

[10] Platonov S.S., On some problems in the theory of the approximation of functions on compact homogeneous manifolds, Sb. Math. 200 (2009), 845-885.

[11] Quinto E.T., Injectivity of rotation invariant Radon transforms on complex hyperplanes in $\mathbf{C}^{n}$, in Integral Geometry (Brunswick, Maine, 1984), Contemp. Math., Vol. 63, Amer. Math. Soc., Providence, RI, 1987, 245-260.

[12] Rudin W., Function theory in the unit ball of $\mathbb{C}^{n}$, Classics in Mathematics, Springer-Verlag, Berlin, 2008.

[13] Schaback R., A unified theory of radial basis functions: Native Hilbert spaces for radial basis functions II, J. Comput. Appl. Math. 121 (2000), 165-177.

[14] Schreiner M., Locally supported kernels for spherical spline interpolation, J. Approx. Theory 89 (1997), 172-194.

[15] Szegő G., Orthogonal polynomials, American Mathematical Society, Colloquium Publications, Vol. 23, 4th ed., Amer. Math. Soc., Providence, R.I., 1975.

[16] Thirulogasanthar K., Saad N., Honnouvo G., 2D-Zernike polynomials and coherent state quantization of the unit disc, arXiv:1303.5483.

[17] Torre A., Generalized Zernike or disc polynomials: an application in quantum optics, J. Comput. Appl. Math. 222 (2008), 622-644.

[18] Wünsche A., Generalized Zernike or disc polynomials, J. Comput. Appl. Math. 174 (2005), 135-163.

[19] Ziegel J., Convolution roots and differentiability of isotropic positive definite functions on spheres, Proc. Amer. Math. Soc. 142 (2014), 2063-2077, arXiv:1201.5833. 